\title{
Functional outcomes, morbidity, mortality and fracture healing rates in 58 consecutive geriatric odontoid fracture patients treated with cervical collar or posterior fusion
}

\author{
Authors William Molinari, Oner Khera, William Gruhn, Robert W Molinari \\ Institution Department of Orthopaedics, University of Rochester, Rochester, New York, USA
}

\section{INTRODUCTION}

Controversy exists as to the most effective management option for elderly patients with type II odontoid fractures. Furthermore, outcomes for patients who do not achieve fusion or fracture healing after treatment remain unclear in the literature. The purpose of this study is to evaluate fracture-healing rates, functional outcomes, complications, and mortality associated with rigid cervical collar and posterior fusion surgery (PSF) for the management of geriatric type II odontoid fractures.

\section{METHODS}

Fifty-eight consecutive elderly patients with type-II odontoid fractures were treated by the same fellowship-trained spinal surgeon (RWM) at a level-1 trauma center during an 8-year period. Patients with more than $50 \%$ odontoid displacement were treated with PSF including C1-2 (PSF group, $\mathrm{n}=25$; average age, 80 years). Patients with less than $50 \%$ odontoid displacement were treated with a rigid cervical collar for 12 weeks (collar group, $\mathrm{n}=33$; average age, 83 years).

Chart reviews were performed evaluating patient comorbidities, treatment complications, and mortality rates. At the time of ultimate follow-up, patients had open mouth, flexion and extension x-rays to assess fracture stability and healing. Additionally, functional outcomes were assessed using Neck Disability Index (NDI), analog pain, and satisfaction questionnaire scores.

\section{RESULTS}

At average 14-month follow-up (range, 3-48 months), fracture-healing rates were higher in the operative group ( $28 \%$ versus $6 \%$ ). Of the nonoperative patients, $64 \%$ had mobile nonunion versus $0 \%$ in the operative group. The average mobility of the nonunion was $2.5 \mathrm{~mm}$ (range, $1-12 \mathrm{~mm}$ ). The NDI scores were lower in the nonoperative group (13 versus 18.3), indicating a trend toward less disability in the nonoperative group $(P=.23)$. There was also a trend toward lower analog pain scores, which were lower in the nonoperative group ( 1.3 versus $1.9, P=.26$ ). Satisfaction scores were equally high in both groups (9.1 versus 8.9). Mobile odontoid nonunion was not associated with higher levels of disability or neck pain, and did not affect scores for patient satisfaction. Mortality rate was $12.5 \%$ in the C-collar group and $20 \%$ in the operative group. Complications were higher in the operative group (24\% versus $6 \%$ ) (Table 1, Fig 1). 


\section{CONCLUSIONS}

Rates of type II odontoid facture healing and stability appear to be higher in geriatric patients treated with PSF. Fracture healing and stability did not correlate with improved outcomes with respect to levels of pain, function, and satisfaction. Mortality and complication rates are lower in patients who are treated with a cervical collar and early mobilization.

\section{Table 1 Outcomes.*}

\begin{tabular}{lll}
\hline & PSF group & Collar group \\
\hline Mortality & $20 \%(5 / 25)$ & $12.5 \%(4 / 33)$ \\
\hline Complications & $24 \%(6 / 25)$ & $6 \%(2 / 33)$ \\
\hline Fracture healing & $28 \%(7 / 25)$ & $6 \%(2 / 33)$ \\
\hline Mobile nonunion & $0 \%$ & $67 \%(20 / 30)$ \\
\hline
\end{tabular}

* PSF indicates posterior fusion surgery.
Fig 1 The neck disability index (NDI) scores at final follow-up. PSF indicates posterior fusion surgery.

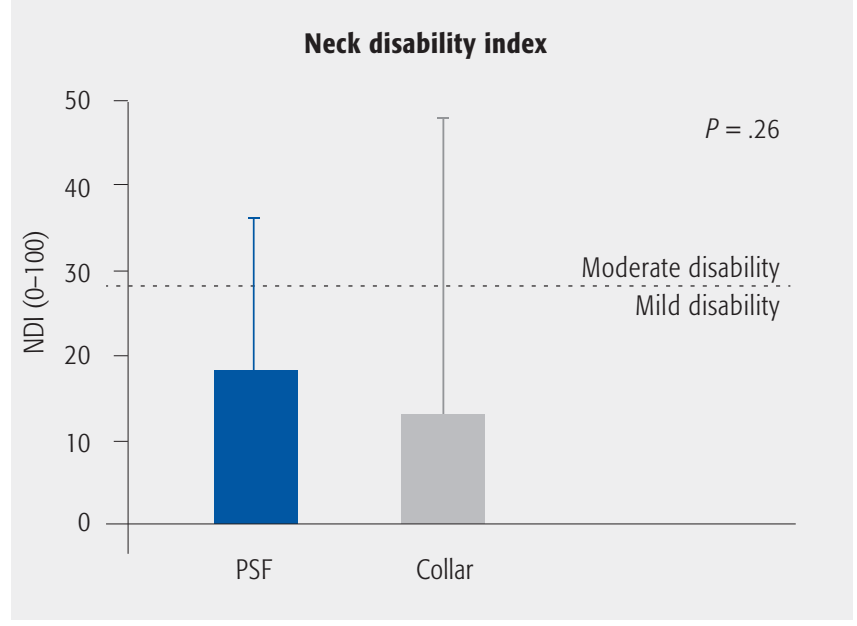

